\title{
A study of diffusion mechanisms in sintering by shrinkage measurements
}

\author{
K M R ACHARI* and R B RAMACHANDER \\ Department of Physics, Osmania University, Hyderabad 500 007, India \\ *Department of Physics, Loyola Academy, Secunderabad 500 010, India \\ MS received 16 December 1986; revised 14 April 1987
}

\begin{abstract}
Shrinkage measurement is important in the study of sintering to ascertain the operative mechanism. Surface area reduction is related to shrinkage with the help of which operative mechanisms at different temperatures are inferred. Densification during sintering is mainly related to volume diffusion, grain boundary diffusion and plastic flow.
\end{abstract}

Keywords. Diffusion mechanism; sintering; shrinkage measurements.

\section{Introduction}

Sintering mechanism in copper has long been disputed. To establish the operative mechanisms at different stages both model (Coble 1961) and real experimental like dilatometric (Akechi and Hara 1979; Masuda and Watanabe 1981) as well as fractographic results (Slesar et al 1982) have been reported. In this paper the fractional shrinkages of copper powder compacts are related to normalized specific surface area reduction and also to the ratio of radius of the neck size to the radius of the particle. From the calculated values of inverse slopes and neck size exponents, the operative mechanisms are reported.

\section{Theory}

The radius of neck curvature $(\rho)$ between two spherical particles is related (Kingery and Berg 1955) to the neck contact area $(x)$ and the radius of the particle $(r)$ as

$$
\rho=x^{2} / 2 r \quad \text { or } \quad x / r=(2 \rho / r)^{\frac{1}{2}} \text {. }
$$

The ratio of $x$ and $r$ is connected to the sintering time $(t)$ (German and Munir 1975) as

$$
(x / r)^{N}=B t,
$$

where $B$ is a constant containing parameters like material transport properties and the particle size, while $N$ represents the characteristic mechanism The above relation can be written as

$$
x / r=(B t)^{1 / N} .
$$

Eliminating $x / r$ from (1) and (3) one obtains

or

$$
2 \rho / r=(B t)^{2 / N}
$$

$$
\rho=(r / 2)(B)^{2 / N} t^{2 / N} \text {. }
$$

For the particles of same size, $r$ is a constant and therefore

$$
\rho \propto t^{2 / N} \text {. }
$$


The relation between the fractional shrinkage $\left(\Delta L / L_{0}\right)$ and $(\rho)$ is deduced (Kingery and Berg 1955) as

$$
\rho / r=\left(\Delta L / L_{0}\right)=x / 2 r^{2}
$$

using (4) and (5)

$$
\rho / r=\left(\Delta L / L_{0}\right)=(B)^{2 / N} / 2 t^{2 / N}
$$

Kingery and Berg (1955) have also obtained another form of relation

$$
\Delta L / L_{0}=k t^{n} \text {, }
$$

which is similar to (6) deduced in its form

where $\quad(B)^{2 / N} / 2=k$ and $2 / N=n$.

So, $\quad \Delta L / L_{0}=k t^{2 / N}$.

If $N$ takes a value 5 , then $n=0.4$ as found experimentally for volume diffusion by Kingery and Berg (1955) for spherical particles i.e.,

$$
\Delta L / L_{0}=k t^{0.4} \text {. }
$$

Hence, we can have a relation

$$
\rho / r=\Delta L / L_{0}=x^{2} / 2 r^{2}=k t^{0.4} .
$$

To evaluate activation energy for volume diffusion (Lee 1972) the relation with fractional shrinkage $f$ used, is

$$
\Delta L / L_{0}=f=\text { const }(t / T) \exp \left(-m Q_{v} / k T\right)
$$

connecting sintering temperature $(T)$, activation energy $\left(Q_{v}\right)$ for volume diffusion and the exponent $m$. Here Lee (1972) assumed that $m=(l / 2 l+1)$ and has taken for spherical particles $l=2$ and got $m=0 \cdot 4$. Lee (1972) experimentally confirmed this exponent by plotting $\ln f$ vs $\ln t$ for constant $T$ and evaluating the slope as 0.4 which agrees with above assumption of $l$ for Niobium. With a view to verify the value of $m$ for copper the authors (Achari et al 1986) have plotted $\ln f$ vs $\ln t$ at constant $T$ and found the value as 0.46 .

Further the normalized specific surface area reduction $\left(\Delta S / S_{0}\right)$ is related as

$$
\Delta S / S_{0}=(x / r)^{m}
$$

In another paper German and Munir (1975) have also expressed

$$
\Delta S / S_{0}=k^{\prime}(B t)^{m^{i} / N} \text {. }
$$

Here $k^{\prime}, m^{\prime}$ and $N$ are constants for a given set of conditions, which are dictated by the controlling mechanism. Examination of (13) reveals that a plot of $\log \left(\Delta S / S_{0}\right)$ vs $\log t$ will give the characteristic value of the inverse slope $N / m^{\prime}$ if a single mechanism is operative. The expected values for each individual mechanism (German and Munir $1975)$ are given in table 1 . Using (5) and (12) the fractional shrinkage is related to $\left(\Delta S / S_{0}\right)$ as

$$
\left(\Delta S / S_{0}\right) \propto(x / r)^{m^{\prime}}=\left[\left(2 \Delta L / L_{0}\right)^{\frac{1}{2}}\right]^{m^{\prime}}
$$


Table 1. Relation between mechanism and $N / m^{\prime}$ (German and Munir 1975).

\begin{tabular}{lccc}
\hline Mechanism & Densification & $\begin{array}{c}\text { Neck size } \\
\text { exponent } N\end{array}$ & $\begin{array}{c}\text { Appropriate } \\
\text { value of } N / \mathrm{m}^{\prime}\end{array}$ \\
\hline $\begin{array}{l}\text { Viscous flow or plastic } \\
\text { flow }\end{array}$ & Yes & 2 & $1 \cdot 1$ \\
Evaporation condensation & No & 3 & $1 \cdot 5$ \\
Volume diffusion & Yes & 5 & $2 \cdot 7$ \\
Grain boundary diffusion & Yes & 6 & $3 \cdot 3$ \\
Surface diffusion & No & 7 & $3 \cdot 5$ \\
\hline
\end{tabular}

and hence

$$
(x / r)^{m^{\prime}}=\left[\left(2 \Delta L / L_{0}\right)^{\frac{1}{2}}\right]^{m^{\prime}}=c k^{\prime}(B t)^{m^{\prime} / n}
$$

where $c$ is another constant.

Here one is left with two alternatives. A plot of

$$
\log \left(\Delta S / S_{0}\right)=\log \left(r_{0}^{2}=r^{2}\right) / r_{0}^{2} \text { vs } \log t
$$

from (13) which yields a value of $N / m^{\prime}$ based on which the operative mechanism can be inferred or a plot of $\log (x / r)$ vs $\log t$ from (14) can be made to yield a value of $N$.

\section{Experimental details}

Copper powder was pressed into pellets using a metallic die. These pellets were sintered for different hours of duration at different temperatures. The initial diameter of the pressed compact is $\left(L_{0}\right)$. The change in diameter $(\Delta L)$ was measured. The fractional shrinkage $\left(\Delta L / L_{0}\right)$ was calculated. The first plot of $\log \left[\left(r_{0}^{2}-r^{2}\right) / r_{0}^{2}\right]$ vs $\log t$ is shown in figure 1. Also the plot of $\log (x / r)=\log \left(2 \Delta L / L_{0}\right)^{\frac{1}{2}}$ vs $\log t$ is shown in figure 2.

\section{Results and discussion}

As expected the plots of (14) and (15) are straight lines for $873 \mathrm{~K}, 973 \mathrm{~K}$ and $1273 \mathrm{~K}$ but not for $1073 \mathrm{~K}$ and $1173 \mathrm{~K}$. For these graphs the reduction in surface area plotted as $\log \left(r^{2}-r_{0}^{2}\right) / r_{0}^{2}$ and $\log x / r$ continuously increases with respect to the sintering duration (figures 1 and 2). The inverse slopes of these lines and the neck size exponents help to confirm the operative mechanisms at the respective temperatures for copper as shown in table 2.

The graphs at $1073 \mathrm{~K}$ and $1173 \mathrm{~K}$ show unexpected deviations. In both the figures, initially the $1073 \mathrm{~K}$ graph is linear. But it bends around the sintering duration of $12600 \mathrm{sec}$, when the grain boundary diffusion terminates as could be seen from the inverse slope in table 2. The slope of second segment confirms the anomalous expansion of copper due to pore coalescence already reported (Schreiner and Tusche 1982). In figure 2 the line at $1173 \mathrm{~K}$ shows a sharp rise of $\log x / r$ compared to other curves. This indicates maximum shrinkage at this temperature for the sample in conformity with volume diffusion (Masuda and Watanabe 1981).

In figure 1 the reduction in surface area for the sample at $1173 \mathrm{~K}$ do not show 


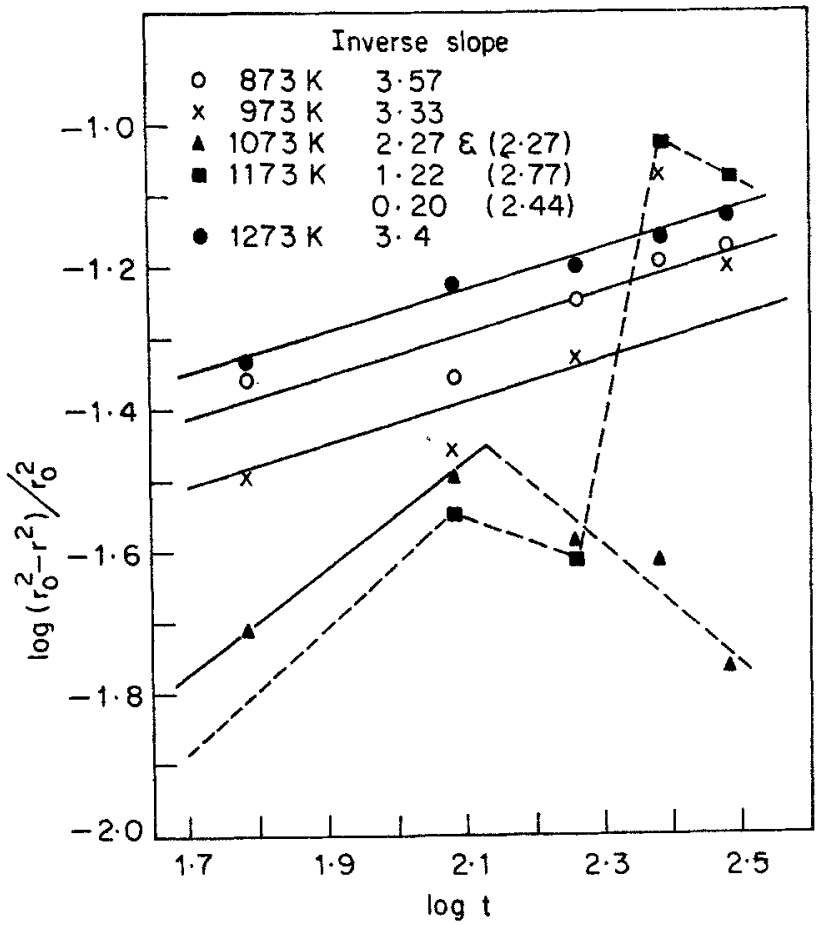

Figure 1. Plot of $\log \left(r_{0}^{2}-r^{2}\right) / r_{0}^{2}$ vs $\log t$.

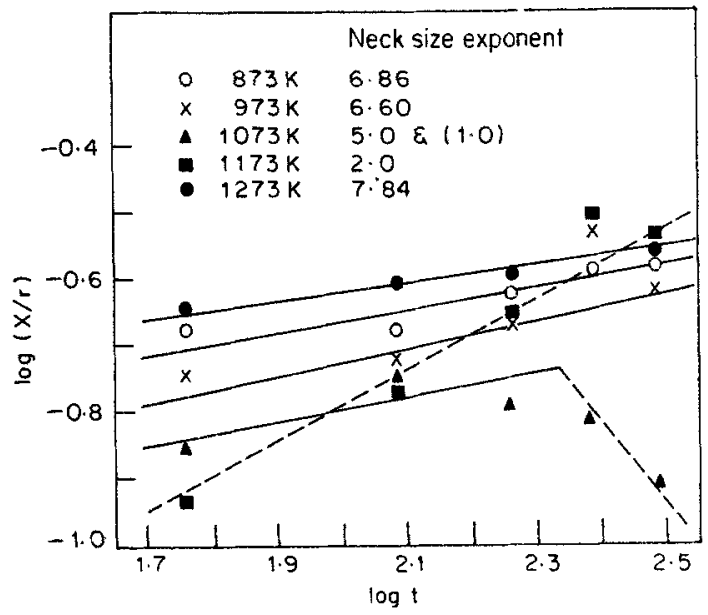

Figure 2. Plot of $\log (x / r)$ vs $\log t$.

continuous increase. It decreases from $72000 \mathrm{sec}$ to $10800 \mathrm{sec}$, as well as from 15400 sec-to $18000 \mathrm{sec}$ of sintering duration. The calculated inverse slopes indicate plastic flow for these regions. But for copper at this temperature volume diffusion is reported to occur (Masuda and Watanabe 1981). We have also observed a maximum densification of the samples (Achari et al 1987) at $1173 \mathrm{~K}$. However the locked-up 
Table 2. Different mechanisms evaluated at different temperatures.

\begin{tabular}{|c|c|c|c|c|}
\hline $\begin{array}{c}\text { Temperature } \\
T(K)\end{array}$ & $\begin{array}{c}\text { Sintering } \\
\text { duration }(\mathrm{sec})\end{array}$ & $\begin{array}{c}\text { Inverse } \\
\text { slope }\left(N / m^{\prime}\right)\end{array}$ & $\begin{array}{c}\text { Neck size } \\
\text { exponent }(N)\end{array}$ & Mechanisms \\
\hline 873 & Total & 3.57 & 6.86 & Surface diffusion \\
\hline 973 & - do- & 3.33 & 660 & Volume diffusion \\
\hline \multirow[t]{2}{*}{1073} & Upto 12600 & $2 \cdot 27$ & $5 \cdot 0$ & $-\mathrm{do}-$ \\
\hline & Beyond & $(2 \cdot 27)$ & $(1 \cdot 0)$ & (Plastic flow starts) \\
\hline \multirow[t]{4}{*}{1173} & Upto 7200 & 1.22 & $2 \cdot 0$ & Plastic flow \\
\hline & 7200 to 10800 & $(2 \cdot 77)$ & - & $\begin{array}{l}\text { (expansion and/or volume } \\
\text { diffusion) }\end{array}$ \\
\hline & 10800 to 15400 & $(0 \cdot 20)$ & - & (Plastic flow) \\
\hline & 15400 to 18000 & $(2 \cdot 44)$ & - & $\begin{array}{l}\text { (Expansion and/or volume } \\
\text { diffusion) }\end{array}$ \\
\hline 1273 & Total & $3 \cdot 4$ & $7 \cdot 84$ & $\begin{array}{l}\text { Volume and/or grain bound- } \\
\text { ary diffusions. }\end{array}$ \\
\hline
\end{tabular}

stresses in the grains, during compacting the powder at high pressures, are released subsequently at $1173 \mathrm{~K}$ while sintering by the plastic flow mechanism (Slesar et al 1982). Further, it is felt that the contribution from volume diffusion perhaps is masked by the plastic flow in the above regions under consideration.

\section{Conclusions}

(i) The fractional shrinkages are related to reduction in surface area and also to the ratio of radius of neck to the radius of the particle. (ii) Anomalous expansion of the sample at $1073 \mathrm{~K}$ confirms pore coalescence. (iii) Plastic flow is suggested along with volume diffusion around $1173 \mathrm{~K}$. (iv) The effect of sintering duration on shrinkage is related to different operative mechanisms.

\section{Acknowledgements}

The authors are thankful to Prof. Anwarur Rahman, Department of Physics, Osmania University and to Mr V Raghavender Rao; Mr A D Manohar and Mr G Sundara Sarma for their assistance while carrying out the work at DMRL.

\section{References}

Achari K M R, Reddy M B and Ramachander R B 1986 J. Mater. Sci. (Communicated) Achari K M R, Reddy M B and Ramachander R B 1987 J. Mater. Sci. (Communicated) Akechi K and Hara Z 1979 Sintering-New developments (ed) M M Ristic (Amsterdam: Elsevier) Coble R L 1961 J. Appl. Phys. 32787

German R M and Munir Z A 1975a Mater. Sci. Res. 10249

German R M and Munir Z A 1975b Mater. Sci. Res. 10259

Kingery W D and Berg M 1955 J. Appl. Phys. 201205

Lee D N 1972 Mater. Sci. Res. 6261

Masuda Y and Watanabe R 1981 Sci. Sintering 13117

Schreiner H and Tusche R 1982 (Proc. 25th Int. Round Table Conference on Sintering)

Slesar M, Besterci M and Dudrova E 1982 Sci. Sintering 1421 